\title{
Focus groups to inform a nutrition intervention for career firefighters
}

Carolyn M Muegge ${ }^{1 *}$, Vanessa M Kleinschmidt ${ }^{1}$, Kara A Johnson ${ }^{1}$, Mercedes Sotos-Prieto ${ }^{2,3,4}$, Steven M Moffatt ${ }^{1}$, Elizabeth A Beverly ${ }^{4,5}$, Maria Korre $^{2}$ and Stefanos N Kales ${ }^{2}$

${ }^{1}$ National Institute for Public Safety Health, Indianapolis, Indiana 46250, USA

${ }^{2}$ Department of Environmental Health, Harvard T.H. Chan School of Public Health, Boston, MA 02115, USA

${ }^{3}$ Department of Food Science and Nutrition, School of Applied Health Sciences and Wellness, Ohio University, Athens, OH 45701, USA

${ }^{4}$ The Diabetes Institute, Ohio University, Athens, $\mathrm{OH} 45701$, USA

${ }^{5}$ Department of Family Medicine, Ohio University Heritage College of Osteopathic Medicine, Athens, OH 45701, USA

\begin{abstract}
Background: Obesity is associated with cardiovascular disease, cancer and ill health among firefighters. Poor diet and firehouse traditions likely contribute to this problem. Worksite health promotion programs could positively affect firefighter health.

Objective: To assess firefighter and firefighter partner perceptions of food, nutrition behavior and culture to inform the Feeding America's Bravest: Survival Mediterranean Style, a Diet-Based Intervention to change Firefighters' Eating Habits.

Materials and methods: Focus group sessions ( 2 firefighter, $n=15$ and 2 partner, $n=17$ ) were conducted to assess attitudes regarding food and nutrition behaviors. A purposeful sampling process was used to ensure diversity in sex, geographic distribution, and length of service. Focus group transcripts were analyzed using content and thematic procedures with triangulation by content experts from different disciplines.
\end{abstract}

Results: Firefighter themes were (1) firehouse eating behaviors are rich in structure and tradition, (2) perceived motivators and challenges to dietary change, and (3) preferences for learning platforms and incentives. Partner themes were (4) eating behaviors at home are loosely structured and driven by family food preferences, (5) partners' perceived motivators and challenges to dietary change, and (6) partners' preferences for learning platforms and incentives.

Conclusions: This study provides evidence that the design of fire service nutrition interventions should consider the cultural expectations of the fire service and firefighter work and family schedules. Educational resources need to target affordable and convenient meal planning.

\section{Introduction}

Obesity is associated with the clustering of cardiovascular disease risk factors among firefighters [1]. Evidence suggests that obesity is increasing in the fire service [2]. This accompanied with the traditional American diet is strongly linked to cardiovascular events [2-3], cancer and other health issues [4,5] among firefighters. A routine diet of fast food, sugary drinks [6], refined carbohydrates and saturated fats [6$8]$, along with firehouse traditions of large portion sizes and second helpings are major contributors to these problems [9].

Feeding America's Bravest: Survival Mediterranean Style is a dietbased intervention to change firefighters' eating habits. It was proposed and funded as a cluster-randomized, controlled health promotion trial designed to test the efficacy and persistence of a Mediterranean Dietbased nutrition intervention in a large fire department [10]. Given the limited amount of data available on firefighter attitudes and beliefs toward nutrition, we conducted focus groups to help design tools and methods to deliver this intervention $[11,12]$.

\section{Materials and Methods}

A purposeful sample of firefighters and firefighter partners (partners) were recruited from the Indianapolis Fire Department to ensure a mix by household, sex, firehouse, shift, and length of service using department rosters. Eligible participants were firefighters and partners who prepared or planned at least some meals and never shared a household with someone working in market research, nutrition, wellness or a clinical medical field.

Discussion guides were developed by the research team based on prior research and the proposed intervention. Four semi-structured group sessions ( 2 firefighter and 2 partner), were facilitated by a trained group leader.

Focus groups were recorded and transcribed verbatim. This study was approved by Chesapeake IRB (Study \#Pro00016089). Participants' written informed consent was obtained prior to the start of each discussion. Each participant was given a financial incentive up to $\$ 175$

${ }^{*}$ Correspondence to: Muegge C, Research Scientist, National Institute for Public Safety Health, 6612 E 75th Street, Suite 200, Indianapolis, IN 46250; Tel: (317) 750-8950; E-mail: carolyn.muegge@nipsh.org

Key words: firefighter, focus groups, nutrition, occupational health, worksite health promotion

Received: October 17, 2018; Accepted: October 26, 2018; Published: October 31,2018 
for firefighters and $\$ 150$ for partners for participation and completion of assignments. All focus groups were held in May 2016.

Content analyses were conducted independently by three investigators using recordings. Each investigator identified codes describing categorical themes among open-ended responses [13] then, working together, indexed these categories into themes. Transcripts were coded until saturation was reached. Discrepancies in coding were discussed and resolved. Triangulation by experts from different disciplines (medicine, nutrition, epidemiology, and biobehavioral health) was conducted to ensure all dimensions of the participants' responses were considered [14]. Transferability was supported via rich descriptions and verbatim quotations included in the data [14]. An investigator not involved with the study conducted an external audit to examine the research protocol and evaluate whether the summary findings were supported by the raw data [14]. Transcript identifiers are used with quotations indicating participant number and focus group number.

\section{Results}

Fifteen career firefighters and 17 partners participated in the focus groups (Table 1).

Four operational frameworks affecting food behaviors were integrated throughout the firefighter comments. All firefighters worked a rotating 24 -hour on duty shift followed by a 48 -hour off duty rotation. Many firefighters reported having second jobs. All firefighters said they pay for work meals at the beginning of each duty day. Amounts ranged from $\$ 10-\$ 15$ per day. All firefighters reported rotating cooking assignments (one to three times a month) that included meal-planning and grocery shopping.

Qualitative analysis considering both firefighter and partner perspectives produced six major themes (Table 2). (Theme 1) Eating behaviors at the firehouse differ from home and are rich in structure and tradition. Firefighters reported some choice in food preparation at home, but none in the firehouse unless they were scheduled to cook. Firefighters said they spend about four hours in the firehouse kitchen preparing meals and snacks, compared to one to two hours per day at home. Firefighters described dinner at the firehouse as particularly rich in tradition.

"Everybody sits down, everybody eats together and it's just kind of a sacred time." - FF2-013

Firefighters reported strict meal-times in firehouses; whereas mealtimes at home flexed around family schedules.

"At work, it's pretty much 12:00 and 6:00 [mealtimes]. At the firehouse, meals are pretty serious." - FF1-004

"At home, I eat whenever or whatever I want. You don't care about the time. You just grab something and eat it." -FF1-004

Firefighters also emphasized that firehouse meals must have enough food for large portions, second helpings and leftovers. Leftovers are often eaten after emergency incidents as a way to help firefighters wind down.

“... there could be a fire incident or some sort of other incident at 2:00 in the morning, like 2:00 to 4:00. And you get back to the fire station and you're hungry again, and you're going to go back to the meals that were prepared ..." - FF1-003

Firefighters indicated that meal quality needs to reflect the amount of money contributed per day.

“... if I pay $\$ 10.00$ and you give me SpaghettiOs, that's not going to fly....... I mean, they're expecting you to cook, cook something good and cook enough." - FF1-007

They also reported that dessert was served every day and additional snacks were always available in the firehouse.

"Somebody will get on the intercom. 'Hey, we've got ice cream sandwiches in here.' And a lot of times at the firehouse, 8:00 or 9:00, somebody is yelling on the PA, 'Fresh popcorn.' So, it just seems like it's always there." - FF1-001

“That's my big problem. I eat twice as much at the firehouse." FF2-011

Table 1. Demographic characteristics of firefighters $(n=15)$ and partners $(n=17)$ participating in nutrition focus groups

\begin{tabular}{|c|c|c|}
\hline \multirow{2}{*}{ Characteristic } & \multicolumn{2}{|c|}{ Mean \pm SD or $n(\%)$} \\
\hline & Firefighters & Partners \\
\hline Age & $46.6 \pm 7.3$ & $46.7 \pm 8.8$ \\
\hline Experiencelyears as firefighters & $19.6 \pm 8.1$ & $22.0 \pm 7.5^{\mathrm{a}}$ \\
\hline \multicolumn{3}{|l|}{ Rank } \\
\hline Private & $8(53 \%)$ & $4(24 \%)^{a}$ \\
\hline Lieutenant & $4(27 \%)$ & $7(41 \%)^{a}$ \\
\hline Engineer & $2(13 \%)$ & $4(24 \%)^{a}$ \\
\hline Captain & $1(7 \%)$ & $2(12 \%)^{a}$ \\
\hline
\end{tabular}

apartner

Table 2. Firefighter and partner focus group themes

\begin{tabular}{|l|l|}
\hline Firefighter & Partner
\end{tabular}

Theme 1. Eating behaviors at the firehouse differ from home and are rich in structure and Theme 4. Eating behaviors at home are loosely structured and driven by family food tradition

Theme 2. Motivators and challenges to firefighter dietary change

Motivators: improved health, increased energy levels, improved quality of life, role preferences modeling

Challenges: cost, time, attitudes of others

Theme 3. Firefighter preferences for learning and dietary change

Preferences for learning: short, hands-on interactive sessions or videos

Incentives for dietary change: factors that mitigate cost of healthy foods, support from

co-workers

Theme 5. Motivators and challenges to partner dietary change

Motivators: improved health, increased longevity, feeling better overall, losing weight, improving self-confidence, role modeling Challenges: time, cost, attitudes of others

Theme 6. Partner preferences for learning and dietary change

Preferences for learning: hands-on sessions, videos, independent study

Incentives for dietary change: factors that mitigate cost of healthy foods 
Firefighters reported that meal planning in the firehouse was based on food costs, grocery store sale items and the daily budget.

“...you can cook healthy but cooking healthy is more expensive when you talk about fresh ingredients, and all that, so you're looking at a lot of fattier cuts of meats, more processed canned foods." - FF2-009

Food choices at the firehouse were dependent on what the cook prepared for the day.

“...for 24 hours, it's like I may be at the mercy of what Bob wants to cook unless I want to bring my own meal." - FF2-009

At home, second jobs and family schedules influenced firefighters' decisions about what to eat.

"I've got a 15-year-old son, he's involved in a lot of sports, and I'm sure anyone that's got kids that they've got activities that kind of dictates when you're eating, where you're eating, what you're eating." FF2-009

The second major theme found among firefighter comments detailed the motivators and challenges to dietary change (Theme 2). Firefighters identified improved health, energy levels, longevity, and quality of life as motivators to improve their diet. They also reported that poor results on an annual department medical exam could serve as a stimulus for change.

"You go to your physical and they say, 'No, your BMI is this, your blood pressure is that.' You wake up and go, 'Oops. I need to correct something." - FF1-007

Multiple firefighters cited a desire to be a good role model for family members as a key reason to adopt a healthier diet.

"I think the other thing too is anybody that has children, that's a big deal because.... they pick up on your eating habits, and what you cook, and if they're exposed to healthier foods, that's setting them up for more success down the road." - FF2-009

However, firefighters perceived the cost of healthy food and the time required to prepare it as challenges to a healthy diet. Other people, at home and in the firehouse, were identified as barriers to healthy eating.

"In my household, it's others, also. Others' attitudes, what they will eat and what they won't." -FF1-005

While on duty, firefighters said that those who tried to pack a healthy meal and eat separately from their shift faced scrutiny.

"Any time in the firehouse, if you're doing something a little different - if somebody just notices a little something off, or if you cook something and it might be fine, but they notice something different about it--" - FF2-010

“--Like sharks on a minnow--" - FF2-013

They also reported concerns about the credibility of a new eating plan. They wanted to know why a particular eating plan was healthy and who made the determination.

"Why is it better than paleo or low carb or whatever. Who says?" - FF2-011

"I don't know, I do my homework before I jump. I’m skeptical..." - FF2-008

Firefighters displayed a strong wariness of fad diets and expressed frustration at what they viewed as ever-changing nutrition guidelines.
"Because, in life, it seems like everything is a fad. You have an Atkins diet, you have a Paleo diet, you have a - they're all come and go, and this one is good for you, and then you find out that it was actually bad for you." - FF1-004

“...they're constantly contradicting themselves. Egg whites are bad, or egg yolks are bad, egg yolks are good, butter is bad, butter is great. It just goes back and forth, so what do you do?" - FF2-008

Overall, firefighters expressed the need for a reason or clearly defined goal to make a change.

"Just, you know, what's the ultimate goal? If I have a decent blood work and don't have any current medical issues, what are you trying to sell me on? What can I gain out of this whole diet?" - FF1-003

Firefighters favored a gradual and integrated change into a new eating plan over abrupt or extreme dietary changes.

The final theme identified from firefighter comments centered on preferences for learning and incentives for dietary change (Theme 3). Firefighters narrowed their preference for nutrition education to short, hands-on, interactive learning sessions or educational videos. Firefighters were not interested in reading materials or intensive learning experiences.

Firefighters felt that factors offsetting the cost of healthier foods were positive incentives to change. Firefighters were interested in coupons for healthy foods, but preferred electronic discounts that could be loaded to a store card. Other incentives centered on work-life at the firehouse. Firefighters indicated it would be easier to develop a new healthy eating plan if everyone at their firehouse was committed to changing together.

The topics for partner focus groups were the same as firefighters. Partners reported that eating behaviors at home are loosely structured and driven by family food preferences (Theme 4). Partners' planning and food decisions revolved primarily around family schedules, including the firefighter's work schedule. Firefighters and partners identified the partner as the primary meal planner, grocery shopper and cook. Partners indicated they often "grab meals on the go," but keep food staples and standard meal ingredients in the house.

Partners said they were more likely to plan meals for several days, whereas firefighters tended to plan for that day's meals only. Cooking time for partners also varied by the shift day. Some partners reported not cooking at all when their partner firefighter was working, while others reported spending up to four hours a day in the kitchen, especially if there were kids in the home.

“...I'm in the kitchen maybe three times out of a week. I don't cook if he's not there. I have no one else to cook for than myself so I find other little things, a salad or something..." - P1-003

Partners reported making decisions based on the preferences of everyone in the household.

"We polled the kids when the kids were little and we said, give us a whole bunch of all the things that you guys like to do and we'll just put in the menu." - P1-006

Partners characterized meals at home as flexible, with loosely planned dinners for the week that were largely dictated by the foods in the house and the family's schedule for that day.

"It depends on the weather. If we're going to grill or if we need to just bake something inside or on the skillet or what we have going on, 
how late he's going to be working his second job, or if I am at work and I get home late." - P2-013

Partners agreed that firefighters eat differently at home compared to work. They reported that firefighters eat less, snack less, and eat fewer desserts at home compared to in the firehouse. Partners characterized firehouse eating patterns as constant eating and snacking on foods containing large amounts of butter and saturated fats.

"...the difference for him is that he snacks more at the firehouse than he does at home." - P1-004

Like firefighters, partners identified motivators and challenges to dietary change (Theme 5). Partners frequently listed improvements in health for themselves and the firefighter, increased longevity, and feeling better overall as motivators. Unlike firefighters, partners reported motivators involving losing weight, looking good for their partner, and improving their self-confidence.

"I want my significant other to like what she sees when she's with me." - P2-010

Partners were also motivated to improve their diets in order to be a good role model for the family.

“...just looking at my kids because I want to make sure that they can see that their mom takes care of herself." - P2-017

Partners identified time and cost as barriers to eating healthy. Partners felt that eating a healthy diet was more expensive and required too much time to sustain in their busy schedules. Similar to firefighters, partners described others, especially kids, as barriers to eating healthy.

“...because my kids don't always like to eat healthy. So sometimes we'll have to make them something else. And the chicken nuggets look really good when I'm still having to make my own lunch, and I'm hungry. So I just grab a chicken nugget." - P2-013

Some partners described the firefighter's work schedule as a barrier to healthy eating for the entire family.

"Oh, yeah. On the days he's on duty, mine is like if he's on duty on a day that I'm home all day, I will eat crap all day because I'm running errands. I'm gonna go through a drive through. I'm gonna have

$$
\text { popcorn for dinner." - P1-009 }
$$

Partners agreed that mitigating the cost of healthy food through discounts and coupons would be an incentive to adopt a healthier eating plan (Theme 6). They reported a preference for hands-on and video instruction for learning but were also open to independent studying or reading.

\section{Discussion}

Consistent with prior findings [6], the current focus group study found firefighters eat better at home than in the firehouse. The present study suggests that firehouse culture creates the differences in eating behaviors between work and home. Firefighters described traditions that included structured mealtimes, unhealthy comfort foods (highcalorie, -fat, and -carbohydrate), large portion sizes, second helpings and leftovers, and desserts and snacks that lead to a poor diet. Firefighters reported that firehouse rules dictate cooking assignments and the dollar amount each firefighter contributes for daily shift meals. While each firefighter is required to pay, the assigned cook is responsible for planning, shopping and preparing the meals for the day. Firefighters not cooking lack choices about meal content, and the culture encourages every firefighter to eat whatever is prepared. Firefighters and their partners generally agree on at-home meal planning, how they prefer to learn, what motivates them to have a healthy diet and challenges to making positive changes.

Previous qualitative research among firefighters has highlighted occupational barriers to healthy eating that include cost [15], portion sizes $[9,16]$, limited food choice $[9,15]$, and firehouse tradition and associated cultural norms $[9,16,17]$. However, such studies targeted eating behaviors at the firehouse and tended to occur within the context of a larger, general conversation about firefighter health. This research targeted nutrition behaviors, attitudes, and knowledge of career firefighters at work and at home and included the partners of firefighters. Additionally, preferred learning styles were assessed to inform a nutritional intervention in the fire service.

\section{Limitations}

These findings represent the perceptions of two small samples of firefighters and firefighter partners from a large fire department in Indiana targeted for a worksite nutrition intervention. Individuals who agreed to participate may have been more health-conscious, interested in nutrition, and ready for change than the average firefighter or partner. Participants were also financially incented to participate.

Despite these limitations, the data collected were rich, informative, and invaluable to the development of the worksite nutrition intervention for firefighters. The targeted and in-depth conversation on nutrition knowledge, attitudes and beliefs and preferred styles of learning from both the firefighters and partners helped guide the development of the intervention. Given that the Mediterranean diet does not require calorie counting, does not explicitly limit portion size, eliminate or prohibit any food and is not a fad, but rather a set of history-based traditions that are backed by a robust evidence-base [18]; the current qualitative research and prior survey research [19] support its use in fire service interventions. These interventions should take into account key points brought forth by firefighters and partners in these conversations.

- Nutrition interventions implemented in the fire service must account for the unique fire house culture and the rich traditions surrounding mealtime.

- Firefighters and partners reported barriers to healthy eating included cost of food, time for preparation, and fit into family and work schedules. Having clearly defined goals that firefighters perceive as improving their quality of life may incent the adoption of a behavior to a lifestyle.

- Firefighters and partners are family-focused; therefore, interventions should include components that engage families in nutritional decision-making.

\section{Acknowledgements}

The authors acknowledge the study's Advisory Boards, Indianapolis Fire Department (IFD) and Indianapolis Local 416 support, the National Fire Organizations (International Association of Fire Fighters, National Volunteer Fire Council, National Fallen Firefighters' Foundation, The Fire Protection Research Foundation, and International Association of Fire Chiefs) that support the research and also the firefighters and their families for their participation.

\section{Funding}

This work was supported by the US Department of Homeland Security [grant number EMW-2014-FP-00612]. 


\section{References}

1. Soteriades ES, Hauser R, Kawachi I, Liarokapis D, Christiani DC, et al. (2005) Obesity and Cardiovascular Disease Risk Factors in Firefighters: A Prospective Cohort Study. Obes Res 13:1756-1763. [Crossref]

2. Soteriades ES, Smith DL, Tsismenakis AJ, Baur DM, Kales SN (2011) Cardiovascular disease in US firefighters: a systematic review. Cardiol Rev 19: 202-215. [Crossref]

3. Geibe JR, Holder J, Peeples L, Kinney AM, Burress JW, et al. (2008) Predictors of On-Duty Coronary Events in Male Firefighters in the United States. Am J Cardiol 101: 585-589. [Crossref]

4. Korre M, Smith D, Kales SN (2018) Obesity and health in the North American Fire Service: research points the way to positive culture change. Occup Med 68: 160-162. [Crossref]

5. Lauby-Secretan B, Scoccianti C, Loomis D, Grosse Y, Bianchini F, et al. (2016) Body fatness and cancer-viewpoint of the IARC Working Group. N Engl J Med 375: $794-$ 798. [Crossref]

6. Yang J, Farioli A, Korre M, Kales SN (2014) Modified Mediterranean Diet Score and Cardiovascular Risk in a North American Working Population. PLoS ONE 9: e87539. [Crossref]

7. Lowden A, Moreno C, Holmbäck U, Lennernäs M, Tucker P (2010) Eating and shift work - effects on habits, metabolism and performance. Scand J Work Environ Health 36:150-162. [Crossref]

8. Esquirol Y, Bongard V, Mabile L, Jonnier B, Soulat JM, et al. (2009) Shift work and metabolic syndrome: respective impacts of job strain, physical activity, and dietary rhythms. Chronobiol Int 26: 544-559. [Crossref]

9. Jahnke SA, Poston WS, Jitnarin N, Haddock CK (2012) Health concerns of the US fire service: perspectives from the firehouse. Am J Health Promot 27: 111-118. [Crossref]
10. Sotos-Prieto M, Cash SB, Christophi CA, Folta S, Moffatt S, et al. (2017) Rationale and design of feeding America's bravest: Mediterranean diet-based intervention to change firefighters' eating habits and improve cardiovascular risk profiles. Contemp Clin Trials 61:101-107. [Crossref]

11. Morse JM, Field PA (1995) Qualitative Research Methods for Health Professionals. 2nd ed. Thousand Oaks: Sage Publications, Inc.

12. Krueger R, Casey M (2000) Focus Groups: A practical guide for applied research. 2nd ed. Thousand Oaks: Sage Publications, Inc.

13. Krippendorf K, (2004) Content Analysis: An Introduction to Its Methodology. 2nd ed. Thousand Oaks: Sage Publications, Inc.

14. Lincoln Y, Guba E (1985) Naturalistic Inquiry. Newbury Park: Sage Publications, Inc.

15. Frattaroli S, Pollack KM, Bailey M, Schafer H, Cheskin LJ, et al. (2013) Working inside the firehouse: developing a participant-driven intervention to enhance healthpromoting behaviors. Health Promot Pract 3:451-458. [Crossref]

16. Dobson M, Choi B, Schnall PL, Wigger E, Garcia-Rivas J, et al. (2013) Exploring occupational and health behavioral causes of firefighter obesity: a qualitative study. $\mathrm{Am}$ J Ind Med 56: 776-790. [Crossref]

17. Staley JA, Weiner B, Linnan L (2011) Firefighter fitness, coronary heart disease, and sudden cardiac death risk. Am J Health Behav 35: 603-617. [Crossref]

18. Martínez-González MÁ, Hershey MS, Zazpe I, Trichopoulou A (2017) Transferability of the Mediterranean Diet to Non-Mediterranean Countries. What Is and What Is Not the Mediterranean Diet. Nutrients 9: E1226. [Crossref]

19. Yang J, Farioli A, Korre M, Kales SN (2015) Dietary preferences and nutritional information needs among career firefighters in the United States. Glob Adv Health Med 4:16-23. [Crossref]

Copyright: (C2018 Muegge CM. This is an open-access article distributed under the terms of the Creative Commons Attribution License, which permits unrestricted use, distribution, and reproduction in any medium, provided the original author and source are credited. 